\title{
Galactocentric distance of the Sun derived from kinematic data
}

\author{
Zi Zhu ${ }^{1,2}$ and Ming Shen ${ }^{3}$ \\ ${ }^{1}$ School of Astronomy and Space Science, Nanjing University, Nanjing 210093, China \\ email: zhuzi@nju.edu.cn \\ ${ }^{2}$ Key Laboratory of Modern Astronomy and Astrophysics (Nanjing University), \\ Ministry of Education, Nanjing 210093, China \\ ${ }^{3}$ National Astronomical Observatories, Chinese Academy of Sciences, Beijing, China \\ email: shenming@nao.edu.cn
}

\begin{abstract}
Based on radial velocity data and Hipparcos proper motions, we present a new determination of the Galactocentric distance based on a purely kinematic model. We have selected three subgroups of Galactic thin-disk components (O-B5 stars, classical Cepheids and Galactic open clusters) to trace the local structure and kinematics of the Galactic disk. Adopting the approximation of axisymmetric circular rotation, we have derived the Sun's distance to the Galactic Center, $\mathrm{R}_{0}=8.25 \pm 0.79 \mathrm{kpc}$ based on O-B5 stars, $\mathrm{R}_{0}=7.98 \pm 0.79 \mathrm{kpc}$ based on Galactic Cepheids and $\mathrm{R}_{0}=8.03 \pm 0.70 \mathrm{kpc}$ using open clusters, all of which are in excellent agreement with the current-best estimate of the Galactocentric distance.
\end{abstract}

Keywords. Galaxy: disk, Galaxy: fundamental parameters, Galaxy: kinematics and dynamics, solar neighborhood

\section{Observational data}

Three separate data sets, namely O-B5 stars, classical Cepheids and Galactic open clusters, will be used for our analysis of the local kinematic structure and to derive the Galactocentric distance.

\section{1. $O-B 5$ stars}

The astrometric data are taken from the revised Hipparcos Catalogue compiled by van Leeuwen (2007), including proper motions and trigonometric parallaxes. The radial velocity data are taken from the General Catalogue of Mean Radial Velocities: 1190 O-B5 stars were selected for our analysis. All of these are single stars listed in the Hipparcos Catalogue; stars belonging to Gould's Belt have been individually excluded.

\subsection{Open clusters}

The data are based on two catalogues: the Catalog of Open Cluster Data (COCD) compiled by Kharchenko et al. (2005), and the new catalog of optically visible open clusters and candidates compiled by Dias et al. (2006). We selected 301 clusters with measurements of heliocentric distances, mean proper motions, mean radial velocities, ages, etc.

\subsection{Galactic Cepheids}

Cross-linking radial-velocity and photometric data of Cepheids in previous studies by several different authors, we selected 215 Galactic Cepheids with complete radial-velocity and photometric data from 249 sample stars in the Hipparcos Catalogue. Since the observing period covering the Hipparcos data was relatively short, we rejected a number 
of suspect binaries. The zeropoint of the period-luminosity relation, $\rho=-1.37 \pm 0.07$, is given by Zhang \& Zhu (2009), derived from Hipparcos parallaxes and photometric measurements of Cepheids.

Figs 1 and 2 show distributions of the selected data projected onto the Galactic plane.

\section{Models}

\subsection{Proper motions}

Assuming that the Galaxy's rotation is axisymmetric, the observed proper motions of objects should represent Oort's model of differential rotation. To first-order approximation we have

$$
\begin{gathered}
\kappa \mu_{\ell} \cos b=\left(u_{0} \sin \ell-v_{0} \cos \ell\right) / r+A \cos 2 \ell \cos b+B \cos b ; \\
\kappa \mu_{b}=\left(u_{0} \cos \ell \sin b+v_{0} \sin \ell \sin b-w_{0} \cos b\right) / r-\frac{1}{2} A \sin 2 \ell \sin 2 b,
\end{gathered}
$$

where the constant $\kappa=4.74047$. Proper motions are expressed in mas $\mathrm{yr}^{-1}$ and the heliocentric distances, $r$, are in units of kpc. The parameters $u_{0}, v_{0}$ and $w_{0}$ are components of the solar motion, pointing to the Galactic Center, in the direction of Galactic rotation, and to the North Galactic Pole, respectively. The Oort constants $A$ and $B$ are defined as

$$
\begin{aligned}
A & =-\frac{1}{2} \mathrm{R}_{0}(\mathrm{~d} \Omega / \mathrm{d} R)_{0} \\
B & =-\Omega_{0}-\frac{1}{2} \mathrm{R}_{0}(\mathrm{~d} \Omega / \mathrm{d} R)_{0},
\end{aligned}
$$

where $\Omega$ is the angular velocity, the subscript ' 0 ' refers to the Sun and $r$ is the distance between the object of interest and the Sun.

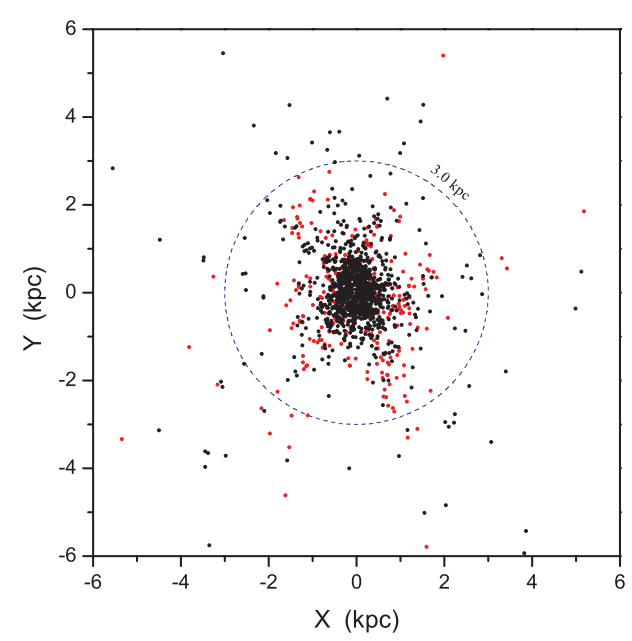

Figure 1. Distributions of 1190 O-B5 stars and 301 open clusters with respect to the Galactic plane. Black dots mark the O-B5 stars, red dots the open clusters. The blue dashed circle marks a heliocentric distance of $3.0 \mathrm{kpc}$. 


\subsection{Radial velocities}

For a star observed at Galactic coordinate $(\ell, b)$ and Galactocentric radius $R$, its radial velocity can be written as

$$
v_{\mathrm{r}}=2 A \mathrm{R}_{0}\left(\frac{\mathrm{R}_{0}}{R}-1\right) \sin \ell \cos b-u_{0} \cos \ell \cos b-v_{0} \sin \ell \cos b-w_{0} \sin b-\delta v_{\mathrm{r}} .
$$

In this equation, $\delta v_{\mathrm{r}}$ expresses a possible systematic offset of the zeropoint defined by the radial velocities, which has been recognized for a long time. Due to the present thin sheets of our samples formed by O-B5 stars, open clusters and Cepheids, the component of the solar motion in the direction of the North Galactic Pole cannot be determined based on radial velocities. In the following analysis of our radial velocity data, $w_{0}$ will be fixed at the value $w_{0}=7.2 \pm 0.4 \mathrm{~km} \mathrm{~s}^{-1}$ of Dehnen \& Binney (1998).

The Galactocentric radius $R$ of a star is given by

$$
R=\left(\mathrm{R}_{0}^{2}+r^{2} \cos ^{2} b-2 r \mathrm{R}_{0} \cos \ell \cos b\right)^{\frac{1}{2}} .
$$

\section{Results}

Using the different subsamples, we derive the Oort constants $A$ and $B$ from proper motions, based on an axisymmetric kinematic model, i.e., Eqs (2.1) and (2.2). Then, by applying least-squares minimization to Eq. (2.5), the parameter $2 A \mathrm{R}_{0}$ can be obtained from the radial velocities. Taking the constant $A$ from the proper-motion solution, the Galactocentric distance $\mathrm{R}_{0}$ can be obtained. Note that the final solution of $\mathrm{R}_{0}$ is obtained in an iterative way. Table 1 shows all kinematic parameters, including the Sun's Galactocentric distance, separately determined from the proper motions and radial velocities of the selected O-B5 stars, open clusters and classical Cepheids.

The present determinations of $\mathrm{R}_{0}$ are based on independent observations of proper motions and radial velocities, derived from three different data sets characterizing the disk component. All results differ from the value of $\mathrm{R}_{0}=8.5 \mathrm{kpc}$ recommended by the IAU in 1985 (Kerr \& Lynden-Bell 1986). Based on a careful statistical analysis, an averaged 'best' value of $R_{0}=8.0 \pm 0.5 \mathrm{kpc}$ was proposed by Reid (1993), using data obtained during

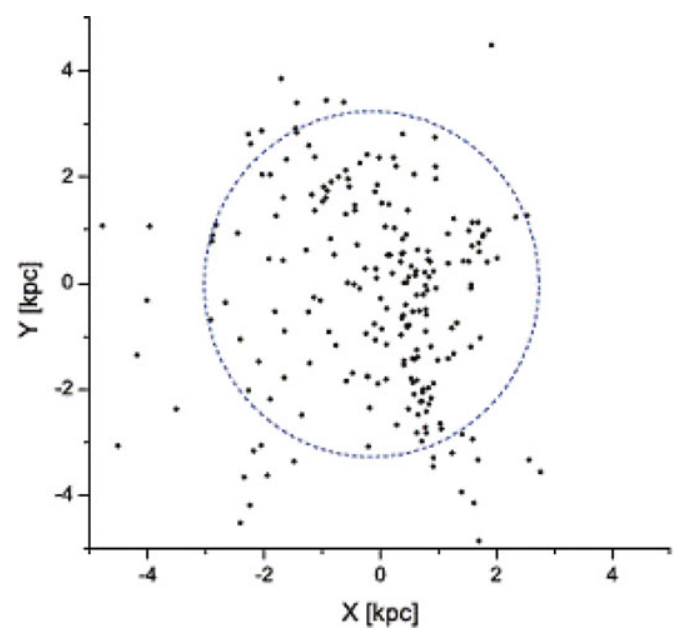

Figure 2. Projected positions of 215 classical Cepheids on the Galactic plane. Most sample stars are found within $3.0 \mathrm{kpc}$ from the Sun. 
Table 1. Galactocentric distance of the Sun and other kinematic parameters obtained from $\mathrm{O}-\mathrm{B} 5$ stars, open clusters and classical Cepheids. The units are $\mathrm{km} \mathrm{s}^{-1}$ for $\delta v_{\mathrm{r}}, 2 A \mathrm{R}_{0}$ and the solar-motion components. The Oort constants are measured in $\mathrm{km} \mathrm{s}^{-1} \mathrm{kpc}^{-1}, \mathrm{R}_{0}$ is expressed in $\mathrm{kpc}$.

\begin{tabular}{lccccccccc}
\hline Sample & Data & $u_{0}$ & $v_{0}$ & $w_{0}$ & $A$ & $B$ & $2 A \mathrm{R}_{0}$ & $\delta v_{\mathrm{r}}$ & $\mathrm{R}_{0}$ \\
\hline \multirow{3}{*}{ O-B5 } & Proper & 10.08 & 9.33 & 5.74 & 14.82 & -14.80 & & & \\
stars & motions & \pm 0.49 & \pm 0.46 & \pm 0.43 & \pm 1.16 & \pm 0.85 & & & \\
\cline { 2 - 11 } & Radial & 9.78 & 13.31 & 7.2 & 14.82 & & 244.4 & 2.69 & 8.25 \\
& velocities & \pm 0.56 & \pm 0.47 & (fixed) & (fixed) & & \pm 13.4 & \pm 0.48 & \pm 0.79 \\
\hline \multirow{3}{*}{ Open } & Proper & 10.82 & 11.41 & 7.75 & 16.16 & -13.19 & & & \\
clusters & motions & \pm 0.69 & \pm 0.70 & \pm 0.52 & \pm 1.07 & \pm 0.76 & & & \\
\cline { 2 - 11 } & Radial & 9.92 & 12.34 & 7.2 & 16.16 & & 259.5 & 2.42 & 8.03 \\
& velocities & \pm 1.24 & \pm 1.18 & (fixed) & (fixed) & \pm 14.7 & \pm 0.86 & \pm 0.70 \\
\hline \multirow{3}{*}{ Classical } & Proper & 12.58 & 14.52 & 8.98 & 17.42 & -12.46 & & & \\
Cepheids & motions & \pm 1.09 & \pm 1.06 & \pm 0.98 & \pm 1.17 & \pm 0.86 & & & \\
\cline { 2 - 10 } & Radial & 5.75 & 12.05 & 7.2 & 17.42 & & 238.5 & 4.21 & 7.98 \\
& velocities & \pm 0.96 & \pm 0.84 & (fixed) & (fixed) & \pm 11.7 & \pm 0.88 & \pm 0.79 \\
\hline
\end{tabular}

the 1970s and 1980s. Avedisova (2005) summarized estimates of $\mathrm{R}_{0}$ derived during the decade prior to their study, and found an average value of $\mathrm{R}_{0}=7.80 \pm 0.33 \mathrm{kpc}$. Analyzing 52 determinations published in the period 1992-2011, Malkin (2012) recommends $\mathrm{R}_{0}=$ $8.0 \pm 0.25 \mathrm{kpc}$ as the current 'best' estimate of the Sun's Galactocentric distance. Our present determinations are consistent with those average values.

\section{Acknowledgements}

This work was funded by the National Natural Science Foundation of China through grants 10973009 and 11173014.

\section{References}

Avedisova, A. S. 2005, Astron. Rep., 46, 435

Dehnen, W. \& Binney, J. 1998, MNRAS, 298, 387

Dias, W. S., Lépine, J. R. D., Bruno, S. A., \& Moitinho, A. 2006, Open clusters and Galactic structure, http://www. astro.iag.usp.br/ wilton

Kerr, F. J. \& Lynden-Bell, D. 1985, MNRAS, 221, 1023

Kharchenko, N. V., Piskunov, A. E., Röser, S., Schilbach, E., \& Scholtz, R. D. 2005, A\&A, 438, 1163

Malkin, Z. 2012, arXiv:1202.6128

Reid, M. J. 1993, ARA\&A, 31, 345

van Leeuwen, F. 2007, $A \mathscr{E} A, 474,653$

Zhang, H. \& Zhu, Z. 2009, Chin. Sci. Bull., 54, 3790 